\title{
The Effect of Facebook Addiction on the Identity Crisis of the University Student
}

\author{
Senouci Boumediene ${ }^{1}$, Djellouli Zineb ${ }^{2}$, M. Yoserizal Saragih ${ }^{3}$ \\ ${ }^{1}$ Educational Measurement and Evaluation, Alger University, Algeria \\ ${ }^{2}$ Counselling and Direction, Oran University, Algeria \\ ${ }^{3}$ Universitas Islam Negeri Sumatera Utara, Indonesia \\ boumediene.senouci@univ-alger2.dz,djellouli.zineb@univ-oran2.dz,yosesaragih77@gmail.com
}

\section{Abstract}

The study aimed to reveal the impact of Facebook addiction on the identity crisis of the university student, and to know the relationship between internet addiction and identity crisis in its various dimensions (concept of self, self-esteem, direction towards the other, school and professional tendencies) of the university student. The study sample consists of (91) university students, and to achieve the objectives of the study two research tools have been used: the first is internet addiction scale developed by 'Ahmed' (2007), and the second is the identity crisis scale developed by the following researchers: 'Shand, Mohammed Samira and Shaheen, Iman Fawzi and Private, Hadi Hassan Raafat' (2015). To reveal the identity crisis and after collecting and processing information statistically using the Statistical Package for Social Sciences (SPSS) and R program, the researchers reached the following conclusions:

- The coefficient of correlation between the concept of self and Facebook addiction is statistically significant.

- The coefficient of self-esteem and internet addiction is statistically non-significant.

- The correlation coefficient between the other direction and internet addiction is statistically non- significant.

- The correlation coefficient between school and occupational tendencies and Internet addiction is statistically significant.

- Internet addiction affects the concept of self.

- Internet addiction does not affect self-esteem.

- Internet addiction does not affect the trend towards the other.

- Internet addiction does not affect school and professional tendencies.
Keywords

facebook addiction; identity crisis, university student

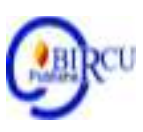

\section{Introduction}

The dynamic developments witnessed in the current era because of the increase in scientific research in various fields helps in the emergence of modern technologies that help humans and facilitate their way of life.

The 21st century has brought other fundamental improvements in communication as well as in information technology. As a consequence, most people nowadays bring portable devices such as laptops, notebooks, ipads, palmtops, smart watches, cell phones, iPads, flash drives, pagers, and so on everywhere they go.(Mana, 2020), this new technology that 21 st has bring effect in communication by bring the Social media.

Social media is an example of a relatively recent development of information technology. (Dahlena \& all, 2020). The Internet is one of the technologies that facilitate communication through social networking sites like Facebook. Through this software we 
can communicate with individuals everywhere. In addition, Facebook user can post photos, videos, open collections, live videos, stories, comments on publications, express opinions and ideas on any topic. This permanent use of Facebook has become a major global public mental health that developed into Facebook addiction.

Undoubtedly, excessive use of Facebook interferes with the real world interaction and communication abilities, while social skills ultimately decrease. Facebook addiction is the new term invented by psychiatrists as its addiction will damage the sleeping habits, health and interest in studies and interaction abilities of real life.(Hasnain \& Chandra Das,2018,p617)

Among all social media applications, Facebook has gained the most popularity and amassed the highest number of users in the world. At the global level, there are over 2.27 billion monthly active users and 1.15 billion daily active users.(Rajesh \& Rangaiah,2020,p1)

From what was presented, it was found that the most commonly used category of Facebook is the student category, which is among the categories characterized by curiosity and love of appearance, imitating others and self-affirmation.

All this if he does not find space, scope and responsiveness in the real world will try to express it in the virtual world through Facebook by carrying out various activities such as building multiple social relationships, monitoring leaflets and comments and a number of links and posts. All this make the individual connected to Facebook for hours in a repeated manner, resulted in what is known as the addiction of Facebook.

From the above we ask the following question:

1. How does Facebook's addiction affect a college student's identity crisis?

From this question, the following sub-questions are branched out:

a. Is there any statistically significant relationship between internet addiction and identity crisis in its various dimensions (self-perception, self-esteem, direction towards the other, school and professional tendencies) at the university student?

b. Is the Internet addiction affects the identity crisis in its various dimensions (selfperception, self-esteem, direction towards the other, school and professional tendencies) at the university student?

\section{1 Hypotheses}

1. There is a statistically significant relationship between internet addiction and identity crisis in its various dimensions (self-perception, self-esteem, direction towards the other, school and professional tendencies) at the university student.

2. Internet addiction affects the identity crisis in its various dimensions (self-perception, self-esteem, direction towards the other, school and professional tendencies) at the university student.

\subsection{Study Objectives}

The current study aims to:

1. Determine how Facebook's addiction affects the university student's identity crisis.

2. Identify the nature of the relationship between internet addiction and the identity crisis in its various dimensions (self-perception, self-esteem, direction towards the other, school and professional tendencies) at the university student.

3. Identify the impact of internet addiction on the identity crisis in its various dimensions (self-perception, self-esteem, direction towards the other, school and professional tendencies) at the university student. 


\subsection{The Importance of Study}

The current study is concerned with one of the problems that resulted from globalization, namely the addiction of Facebook because it is the most widespread problem in many regions of the world including Algeria, especially among the university youth, and how this addiction affects their identity crisis.

\subsection{Procedural Concepts}

1. Internet addiction: The degree which a university student gets by answering the internet addiction scale. Arithmetic medium between $(1-1,6)$ indicates a weak addiction, while the medium between $(1,6-2,2)$ indicates a medium degree of addiction, while the medium between ( 2,2-2,8) Means a high level of addiction.

2. Identity crisis: The degree which a university student recognizes it by answering the id crisis scale, where the higher the student's score on the scale, the more he or she has an identity crisis.

\section{Review of Literatures}

\subsection{Social-Networking Sites and Facebook}

SNS are defined as mobile- or Internet-based social platforms created and designed to enable users to communicate, collaborate and share content across contacts and communities. Many researchers (e.g. García-domingo et al. 2017; Heerden \& Jordaan 2017; Hu, Zhao \& Huang 2015; Lu, Lin \& Lu 2016; Robertson \& Kee 2017) agree on the definition of SNSs suggested by Boyd and Ellison (2008), who defined the term as platforms that allow individual users to: 1- create public and semi-public profiles within circumscribed systems 2- choose other users with whom they can share connections 3observe and navigate through lists of connections and those created by others in the same system.(Gwena \& others,2018,p2).

\subsection{Facebook}

Facebook is a social networking website where users can post comments, share photographs and post links to news or other interesting content on the web, chat live, and watch short-form video.

Facebook is a social networking website where users can post comments, share photographs and post links to news or other interesting content on the web, chat live, and watch short-form video.(Nations,2019)

With over 800 million users, face book is one of the most famous SNS. As much as seventy languages are provided on the website. Recently, with the advent of smart phones, people are always on the go, they $\log$ on to Face book without needing a personal computer and check their profiles. There are almost 475 mobile operators worldwide that are involved in the promotion of face book. According to Facebook Press 2012, presently, 350 million people access face book through their phones.(Mahmood \& Farooq,2014,p56).

\subsection{Identity Crisis}

Among the first to define the name and content of the concept of identity was Erikson, who mostly defined identity as a subconscious process that merges a personality into a single whole and forms links between the individual and the social world.

Erikson argues that identity can be described with the answer to the question "who am I?" He also indicates that identities are not always positive in that sometimes individuals choose a negative identity to maintain their sense of 
integrity. An example would be identifying oneself with a violent person and then becoming a violent person, too.( Dombrovskis,2016,p309).

\subsection{How did Erikson See Identity Confusion Within the Crisis?}

Erikson recalled in Identity: Youth and Crisis that he coined the term identity crisis during the Second World War in his clinical work with soldiers who had Erikson defined identity confusion as "a split of self-images, a loss of center and a dispersion" The symptoms associated with the transition to adulthood include problems of intimacy, distortions of time perspectives (vacillations between a sense of urgency and procrastination), a "diffusion of industry" (an inability to concentrate on required tasks and an excessive preoccupation with some one-sided activity), and a choice of negative identity These symptoms are often transitory, but in its more severe manifestations identity confusion can be observed "[1] in excessively prolonged moratoria ... [2] in repeated impulsive attempts to end the moratorium with sudden choices ... [along with a denial] that some reversible commitment has already taken place, or [3] sometimes in severe regressive pathology" (E. Cote,2018,p p 2-3)

\subsection{Previous Studies}

\section{1. "Kuan-Ying Hsieh \& Ray C. Hsiao \& Yi-Hsin Yang \& Kun-Hua Lee \& Cheng- Fang Yen"(2019)}

Entitled "Relationship between Self-Identity Confusion and Internet Addiction among College Students: The Mediating Effects of Psychological Inflexibility and Experiential Avoidance". The aim of this study was to examine the relationship between self identity confusion and IA and the mediating effects of psychological inflexibility and experiential avoidance (PI/EA) indicators in college students. The sample of the study included 500 college students. The research tools that were used were the Self-Concept and Identity Measure. The results demonstrated that the severity of self-identity confusion was related to the severity of IA, either directly or indirectly. The indirect relationship was mediated by the severity of PI/EA. Self-identity confusion and PI/EA should be taken into consideration by the community of professionals working on IA. Early detection and intervention of self-identity confusion and PI/EA should be the objectives for programs aiming to lower the risk of IA.

\section{2. "Ting-Hsiang Chen \& Ray C. Hsiao \& Tai-Ling Liu \& Cheng-Fang Yen "(2019)}

Entitled "Predicting effects of borderline personality symptoms and self-concept and identity disturbances on internet addiction, depression, and suicidality in college students: A prospective study ". The aims of this study were to evaluate the predicting effects of borderline personality symptoms and self-concept and identity disturbances on internet addiction, significant depression, and suicidality among college students at follow-up assessments conducted one year later. A sample of 500 college students aged between 20 and 30 years participated in this study. Their levels of borderline personality symptoms, self-concept and identity disturbances, internet addiction, depression, and suicidality at baseline and at follow-up interviews were assessed through the Borderline Symptoms List, Self-concept and Identity Measure, Chen Internet Addiction Scale, Beck Depression Inventory-II, and questions related to suicidality from the Epidemiological version of the Kiddie Schedule for Affective Disorders and Schizophrenia, respectively. A total of 324 college students received follow-up assessments one year later. Among them, 15.4\%, $27.5 \%$, and $17 \%$ had internet addiction, significant depression, and suicidality, respectively. The results revealed that severity of borderline symptoms, disturbed identity, 
unconsolidated identity, and lack of identity at initial assessment increased the occurrence of internet addiction, significant depression, and suicidality at follow-up assessment, except for the predictive effect of unconsolidated identity on internet addiction. The results indicated that self-concept and identity and borderline symptoms may have a significant role in the risk of mental health problems in college students.

\section{3. "Touraj Bnirostam \& Hayedeh Saberi "(2017)}

Entitled "Study of Loneliness and Identity Styles among Internet Addicted Students in Faculty of Engineering, Islamic Azad University, Central Tehran Branch ". This study aimed to investigate level of sense of loneliness, identity styles and Internet Problematic Use among students. The sample of the study included all undergraduate students of technical-engineering faculties. The tool that was used was a questionnaire to collect the data according to the descriptive method. After the analyses of data the study showed the following result: The sense of loneliness is higher than two other variance which in turn end in internet addiction and identity disorders.

\section{4. "Sevda Aslan"(2016)}

Entitled "The views of university students regarding internet addiction ". This study aimed to determine the views of university students regarding Internet addiction. The study sample consisted of 64 4th year students studying at Kurıkkale University, Faculty of Arts and Sciences, department of philosophy and Kirikkale University, Faculty of Health Sciences, department of child development, in spring, academic year 2013-2014. In this study, University Students' Views Regarding Internet Addiction Form was developed by the researcher in order to determine the views of university students. These forms consisted of nine open ended questions related to the students' views about Internet addiction. The findings of this research are; not being able to fulfill the requirements of friendship, which are communication, sharing and responsibility; trying to overcome those via social media; being lonely, they have scarcely any family ties, the adolescent considers entering the courses and doing literature review in the library as a waste of time and therefore obtains insufficient academic information solely from the Internet.

\section{5. "Aivis Dombrovskis" (2016)}

Entitled "Identity and an Identity Crisis: The Identity Crisis of First-Year Female Students at Latvian Untversities and Their Sociodemographic Indicators". This study aimed to find links between identity crises and the sociodemographic indicators of firstyear female students at Latvian universities. The research sample consists of $501(\mathrm{~N}=501)$ students aged 18 to $27(\mathrm{M}=20.33$; $\mathrm{SD}=1.937)$. The author used the Identity Distress Survey (IDS) for the research (Berman et al., 2004), as well as the sociodemographic data of the students. The Spearman correlation coefficient was used to analyse links between IDS indicators, and detailed analysis of links between IDS factors was also conducted.

\section{6. "Ali Soleymani \& Mehrzad Farahati" (2014)}

Entitled "The Impact of Excessive Internet Use on Communication Skills and Mental Health in Cafe Internet Users" this study aimed to determine the impact of excessive internet use on communication skills and mental health in adolescents who use internet in the cafe. The research sample consists of 160 customers ( 80 girls and 80 boys) of the internet cafes in Tehran, whose age is between 13 to 23 years. These persons were randomly selected from clients of the Internet cafes. The participants completed the communication skills questionnaire (Harje and Marshal, 1986), General Health 
questionnaire (Goldberg, 1979) and the researcher-developed questionnaires. The results have shown no difference between adolescents' communication skills regarding the use of internet games. However, there was a difference between adolescents' mental health in terms of frequency of using the internet games. Those who spend fewer (less than 6) hours using the Internet games exhibited lesser physical symptoms, reduced level of anxiety and depression and were more socializing.

\section{7. "Hoon Jung Koo \& Jung-Hye Kwon" (2014)}

Entitled " Risk and Protective Factors of Internet Addiction: A Meta-Analysis of Empirical Studies in Korea " A meta-analysis of empirical studies performed in Korea was conducted to systematically investigate the associations between the indices of Internet addiction (IA) and psychosocial variables. Systematic literature searches were carried out using the Korean Studies Information Service System, Research Information Sharing Service, Science Direct, Google Scholar, and references in review articles. The key words were Internet addiction, (Internet) game addiction, and pathological, problematic, and excessive Internet use. Only original research papers using Korean samples published from 1999 to 2012 and officially reviewed by peers were included for analysis. Ninety-five studies meeting the inclusion criteria were identified. The magnitude of the overall effect size of the intrapersonal variables associated with internet addiction was significantly higher than that of interpersonal variables. Specifically, IA demonstrated a medium to strong association with "escape from self" and "self-identity" as self-related variables. "Attention problem", "self-control", and "emotional regulation" as control and regulation-relation variables; "addiction and absorption traits" as temperament variables; "anger" and "aggression" as emotion and mood variables; "negative stress coping" as coping variables were also associated with comparably larger effect sizes. Contrary to our expectation, the magnitude of the correlations between relational ability and quality, parental relationships and family functionality, and IA were found to be small. The strength of the association between IA and the risk and protective factors was found to be higher in younger age groups.

Through presenting the previous studies, we find that most of them have focused on internet addiction. We found that some of them tried to identify the relationship between addiction to the Internet and social communication among the individuals. One study focused on the topic of isolation and another on self-identity. Therefore the current study came out of the scientific gap, that the researchers observed, between internet addiction and its impact on the identity crisis.

\section{Research Methods}

\subsection{Study Procedures}

\section{a. Sample Survey}

The sample of the study consists of (30) students from Saida University, Faculty of Social Sciences selected in a deliberate way. Table 01 below shows the percentage of the sample in term of sex.

Table 1. Sample composition in terms of sex

\begin{tabular}{|c|c|c|}
\hline Sex & Sample & Percentage \\
\hline Male & 6 & $20 \%$ \\
\hline Femelle & 24 & $80 \%$ \\
\hline Total & 30 & $100 \%$ \\
\hline
\end{tabular}


According to the table, the majority of the participants surveyed were female, with a percentage of $80 \%$. The proportion of males is estimated at $20 \%$.

\section{b. Study Tools}

First: The identity crisis scale:

1. Description of the study tool: The researchers used the identity crisis scale by researchers "Shand, Mohammed Samira and Shaheen, Iman Fawzi and Al-Dhaif, 'Hadi Hassan Raafat" (2015).

Here are the steps to find the tool:

a) Determining the goal of the tool: The goal of the tool is to measure the identity crisis of the student at the University of Saida.

b) Sources for the formulation of the paragraphs of the scale: The researchers relied on the study by "Shand, Mohammed Samira et al" (2015) which addressed the psychometric characteristics of the identity crisis scale in adolescents. The scale in its initial form includes (58) items all positive

c) Test correction method: Five alternatives have been identified to answer the scale items which are (strongly ok, OK, neutral-non-OK, not-highly, non-approved) by weights (54-3-2-1), and in the case of negative item the weights are reversed.

2. Symmetric characteristics: To use the tool and assess its psychometric characteristics, validity was used through Facebook consistency and self-validity, while stability was used by Alpha Kornbach and half-fragmentation.

a) Validity

1) The sincerity of internal consistency: The Pearson correlation coefficient was applied to determine the extent to which each item was associated with the overall score of the scale and the results showed retention (29) item as the values of the Pearson correlation coefficient for these item ranged from $(0,304)$ to $(0,657)$, which indicate the correlations of the item for the total sum, and these results indicate consistency. The remaining items of the scale are consistent, indicating that the scale is characterized by internal consistency.

2) Self-validity: Self-validity was extracted through the stability coefficient where the value of the stability coefficient is $(0,889)$ and after calculating the square root of this value it gives the value $(0,942)$, a high value represents self-validity and therefore the tool has high validity.

b) Stability: The value of the stability coefficient of 'Alpha Kornbach' is $(0,889)$. This is a high value indicating that the tool has high stability, while stability by fragmentation filtering was estimated before correction by $(0,564)$ and corrected by the equation of 'Gutman' because the number of item of the scale is (29) and when divided, the two becomes unequal and reaches $(0,716)$ which is an acceptable value that indicates that the tool's stability.

Second: Facebook addiction scale:

1. Description of the study tool: The researchers devlope the Facebook Addiction Scale according to Internet Addiction Scale by Ahmed (2007).

Here are the steps to get the tool:

a) Determining the goal of the tool: The goal of this tool is to measure Facebook addiction among the student at the University of Saida.

b) Sources for the formulation of the items of the scale: The researchers relied on the study by "Boubaaia, Somaya" (2017) which dealt with Facebook addiction and its 
relation to the emergence of sleep disorders in a sample of university youth that relied on 'Ahmed' scale (2007). The scale in its initial form include (36) items all positive.

c) Test correction method: Three alternatives have been developed to answer the scale item which are (apply, perfect to some extent, do not apply) with weights (3-2-1).

2. Validity

a) The sincerity of internal consistency: The Pearson correlation coefficient was applied to determine the extent to which each item was associated with the overall score of the scale and the results showed that the values of the Pearson correlation coefficient between the item and the total range ranged from $(0,376)$ to $(0,857)$, which indicate the correlation of all the items. So it can be said that these results indicate the existence of consistency between the items of the scale, indicating that the scale is characterized by a high level of sincerity.

b) Self-validity: After calculating stability we get a value of $(0,973)$, and since self-honesty is the square root of stability, the value of self-validity then is $(0,986)$, which means that the tool has high validity.

3. Stability: The value of the stability coefficient of 'Alpha Kornbach', which is equal to $(0,973)$, is a high value indicating that the tool has high stability. While stability by fragmentation filtering was estimated before correction by the value $(0,870)$ and corrected by the equation of 'Spearman Brown' because the number of paragraphs of the scale are (36) and when divided they become equal and reached the value $(0,931)$, which is a high value that indicates the instrument's stability.

\subsection{Methods}

The present study, which is the 'impact of Facebook addiction on the university student's identity crisis', requires the use of the descriptive method, since it is appropriate to its nature, purposes and objectives.

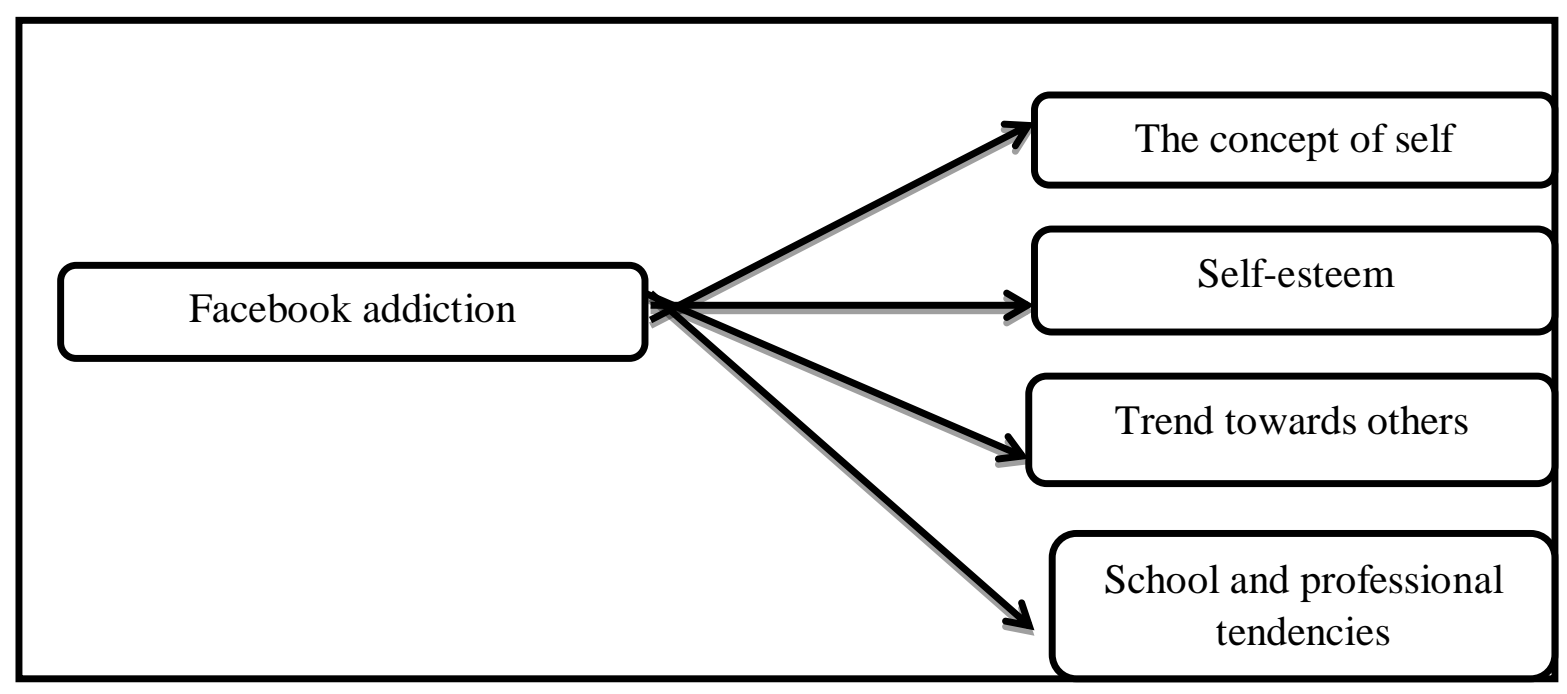

Figure 1. The study model

\section{a. The Study Sample}

The sample of the study includes students from the University of Saida, Faculty of Social Sciences. Table 02 below shows the percentage of the sample in term of sex. 
Table 2. Sample composition in terms of sex

\begin{tabular}{|c|c|c|}
\hline Sex & Sample & Percentage \\
\hline Male & 18 & $19.8 \%$ \\
\hline Femelle & 73 & $80.2 \%$ \\
\hline Total & 91 & $100 \%$ \\
\hline
\end{tabular}

According to the table, the number of males is 18 which represent $19.8 \%$, whereas the number of females is 73 with a percentage of $80.2 \%$. This indicates a variation in the sample.

\section{b. Application Procedures}

The study was applied to a sample of 100 students from Saida University, Faculty of Social Sciences during the academic year 2019/2020. Only 91 forms were retrieved.

\section{c. Statistical Methods}

Table 3. Statistical methods and programs used for data analyses

\begin{tabular}{|c|c|}
\hline Statistical method & The software used \\
\hline Duplicate & SPSS 22 \\
\hline Percentages & \\
\hline Twisting and flattening & \\
\hline Propagation shape & $\mathrm{R}$ \\
\hline Pearson Correlation Coefficient & \\
\hline Regression analysis & \\
\hline
\end{tabular}

\section{Results and Discussion}

\subsection{The Result of the First Hypothesis}

There is a statistically significant relationship between Facebook addiction and identity crisis in its various dimensions (self-perception, self-esteem, direction towards the other, school and professional tendencies) for the university student.

In order to ensure the moderation of the distribution, the twisting and flattening coefficients were calculated. The following results were reached:

Table 4. The coefficients of sprain and flatness of the study variables

\begin{tabular}{|l|l|l|l|l|l|l|}
\hline \multicolumn{2}{|c|}{} & $\begin{array}{c}\text { self- } \\
\text { perception }\end{array}$ & $\begin{array}{c}\text { self- } \\
\text { esteem }\end{array}$ & $\begin{array}{c}\text { direction towards } \\
\text { the other }\end{array}$ & $\begin{array}{c}\text { school and professional } \\
\text { tendencies }\end{array}$ & $\begin{array}{c}\text { internet } \\
\text { addiction }\end{array}$ \\
\hline $\mathrm{N}$ & Valide & 90 & 89 & 89 & 87 & 91 \\
\hline & $\begin{array}{l}\text { Missing } \\
\text { values }\end{array}$ & 1 & 2 & 2 & 4 & 0 \\
\hline \multicolumn{2}{|l|}{ Twisting } &, 794 & 1,341 &, 173 & -357 & 1,191 \\
\hline Flattening &, 779 & 1,937 &,- 419 &,- 062 & 1,461 \\
\hline
\end{tabular}

From the table, it is noted that the twisting coefficients of the dimensions of the identity crisis ranged from 0,357 to 1,341 . While the coefficient of flatness ranged from 0,062- to 0,779 . Which means that the two coefficients are above 0 and do not exceed 3 . In addition, the twisting coefficient of Facebook addiction is 1.191 and its flatness coefficient is 1,461 , these values are also greater than (0) and do not exceed (3). This result show that frequency distribution is moderate. 
Make sure there's a linear relationship through the drawing propagation tables:
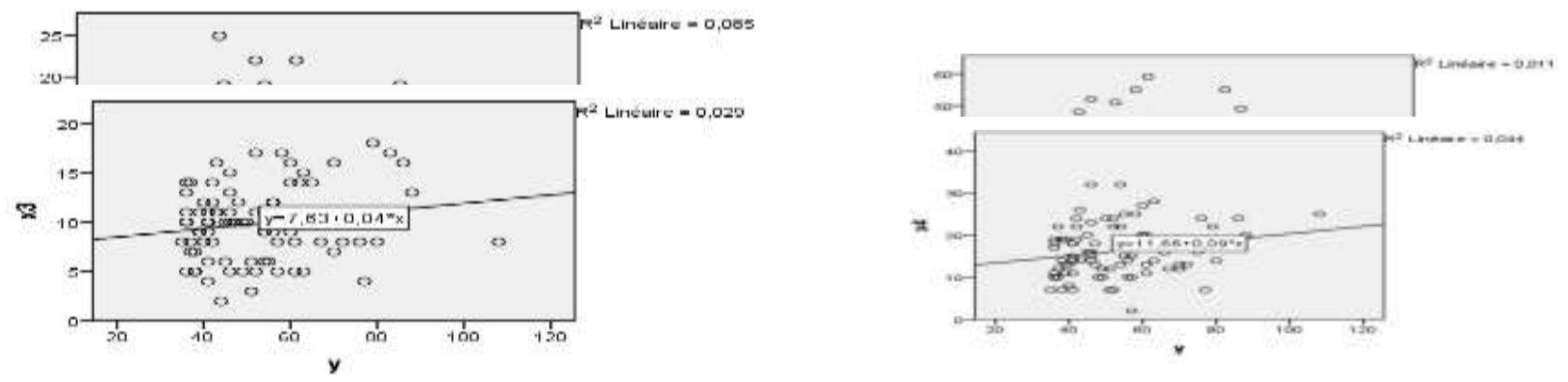

Figure 2. The forms of proliferation between the dimensions of the identity crisis and Facebook addiction

Table 5. Results of Pearson's Correlation Coefficient between Self-Concept and Facebook Addiction

Pearson's product-moment correlation

data: $\mathrm{x} 1$ and $\mathrm{y}$

$\mathrm{t}=2.8507, \mathrm{df}=88, \mathrm{p}$-value $=0.005434$

alternative hypothesis: true correlation is not equal to 0

95 percent confidence interval:

0.089021260 .46956951

sample estimates:

cor 0.2907522

From the results, it is clear that the value of pearson's correlation coefficient between the concept of self and Facebook addiction is 0,29. This indicates that there is a weak relationship between the two variables. In order to ascertain the statistical significance of these results, the following equation will be applied:

$\mathrm{T}=\mathrm{r} \cdot \sqrt{\frac{n-2}{1-r^{2}}}$

$\mathrm{T}=0.290 \times \sqrt{\frac{91-2}{1-(0.290)^{2}}}=0.29 \times \sqrt{\frac{89}{0.9159}}=0,290 \times \sqrt{97,17}$

$\mathrm{T}=0,290 \times 9,85=2,8565$

When we refer to the t-scale at the degree of freedom (89), the scheduled value is estimated at (1.984) and it is smaller than the calculated value $(2,8565)$. Since the calculated $\mathrm{T}$ value is greater than the one given by the table, it can be said that the coefficient of correlation between the concept of self and Facebook addiction is statistically significance. 
Table 6. Pearson's

Correlation Coefficient between Self-Esteem and Facebook Addiction

Pearson's product-moment correlation

data: $\mathrm{x} 2$ and $\mathrm{y}$

$\mathrm{t}=1.0007, \mathrm{df}=87, \mathrm{p}$-value $=0.3197$

alternative hypothesis: true correlation is not equal to 0

95 percent confidence interval:

$-0.10388780 .3080891$

sample estimates:

cor 0.1066769

According to the results, it is clear that the value of Pearson's correlation coefficient between self-esteem and Facebook addiction was (0.10) which indicates a weak relationship between the two variables, and in order to ascertain the statistical significance of these results, the following equation will be applied:

$\mathrm{T}=0.106 \times \sqrt{\frac{91-2}{1-(0.106)^{2}}}=0.106 \times \sqrt{\frac{89}{0.98304}}=0,106 \times \sqrt{99.69}$

$\mathrm{T}=0,106 \times 9,98=1,05788$

When you refer to the t-scale at the degree of freedom (89), the scheduled value is estimated at (1.984) and it is greater than the calculated value (1.05788). Since the calculated $\mathrm{T}$ value is smaller than the one given by the table, it can be said that the coefficient of self-esteem and Facebook addiction is statistically non-significant.

Table 7. Pearson Correlation Coefficient between Trend towards the Other and Facebook Addiction

Pearson's product-moment correlation

data: $\mathrm{x} 3$ and $\mathrm{y}$

$\mathrm{t}=1.6207, \mathrm{df}=87, \mathrm{p}$-value $=0.1087$

alternative hypothesis: true correlation is not equal to 0

95 percent confidence interval:

$-0.038433180 .36638796$

sample estimates:

cor 0.1711938

The value of Pearson's correlation coefficient between self-esteem and Facebook addiction is (0.17) and indicates a weak relationship between the two variables, and in order to ascertain the statistical significance of these results, the following equation will be applied:

$\mathrm{T}=0,171 \times \sqrt{\frac{91-2}{1-(0.171)^{2}}}=0.171 \times \sqrt{\frac{89}{0.970759}}=0,171 \times \sqrt{91.68}$

$\mathrm{T}=0,171 \times 9,57=1,6347$ 
When you refer to the t-scale at the degree of freedom (89), the scheduled value is estimated at $(1,984)$ and is greater than the calculated value $(1,63647)$. Since the calculated $\mathrm{T}$ value is smaller than the one given by the table, it can be said that the correlation coefficient between Trend towards The other and Facebook addiction is statistically nonindicative.

Table 8. Pearson's Correlation Coefficient between School and Professional Tendencies and Facebook Addiction

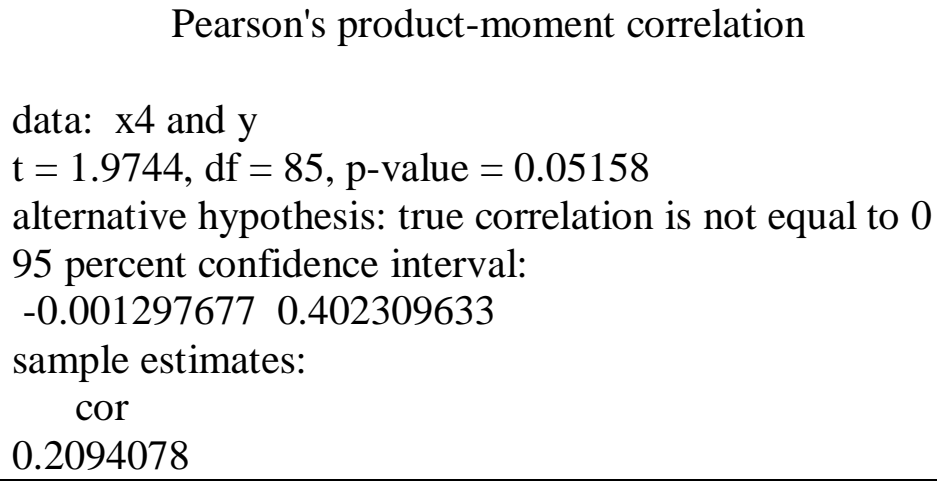

It is clear from the table above, that the value of Pearson's correlation coefficient between self-esteem and Facebook addiction is (0.20) which indicates a weak relationship between the two variables, and in order to ascertain the statistical significance of these results, the following equation will be applied:

$\mathrm{T}=0,209 \times \sqrt{\frac{91-2}{1-(0.209)^{2}}}=0.209 \times \sqrt{\frac{89}{0.956319}}=0,209 \times \sqrt{93.06}$

$\mathrm{T}=0,209 \times 9,64=2,01476$

When you refer to the t-scale at the degree of freedom (89), the scheduled value is estimated at $(1,984)$ and it is smaller than the calculated value $(2,01476)$. Since the calculated $\mathrm{T}$ value is greater than the one given by the table, it can be said that the correlation coefficient between school and occupational tendencies and Facebook addiction is statistically significant.

\subsection{The Result of the Second Hypothesis}

Facebook addiction affects the identity crisis in its various dimensions (selfperception, self-esteem, direction towards the other, school and professional tendencies) of the university student.

In order to test this hypothesis, regression analysis was used and the following results were reached:

Table 9. Regression analysis between the concept of self and Facebook addiction

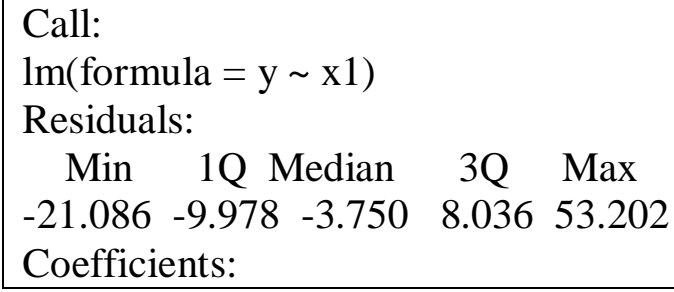




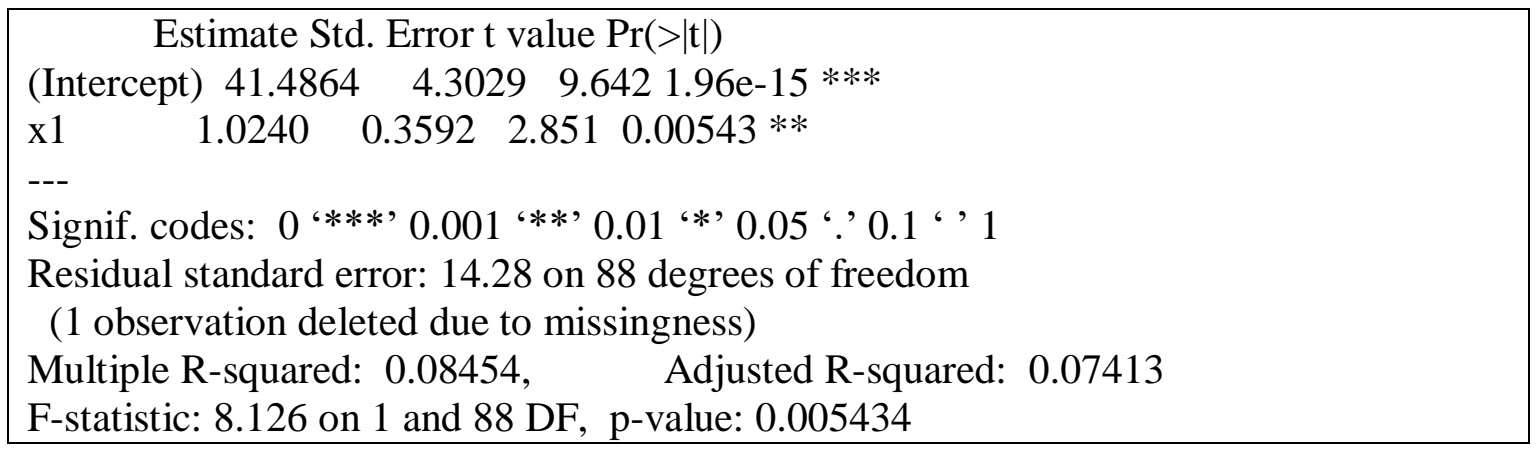

According to the table the value of the correlation coefficient between Facebook addiction and self-concept is 0,08 which represents $8 \%$. It is a weak correlation and the R2 selection factor is 0,07 which represents $7 \%$ only. The change in self-perception is due to Facebook addiction.

It is also noted that the level of significance which is 0,0 is smaller than the level of moral Sig which is equal to 0,05 and this indicates the difference between the concept of self and Facebook addiction. Therefore the alternative hypothesis that Facebook addiction affects the concept of self is accepted. The regression line equation between Facebook addiction and the concept of self can be written as follows:

$\mathrm{Y}=41,4864+1,0240 \times 1$

Table 10. Regression analysis between self-esteem and Facebook addiction

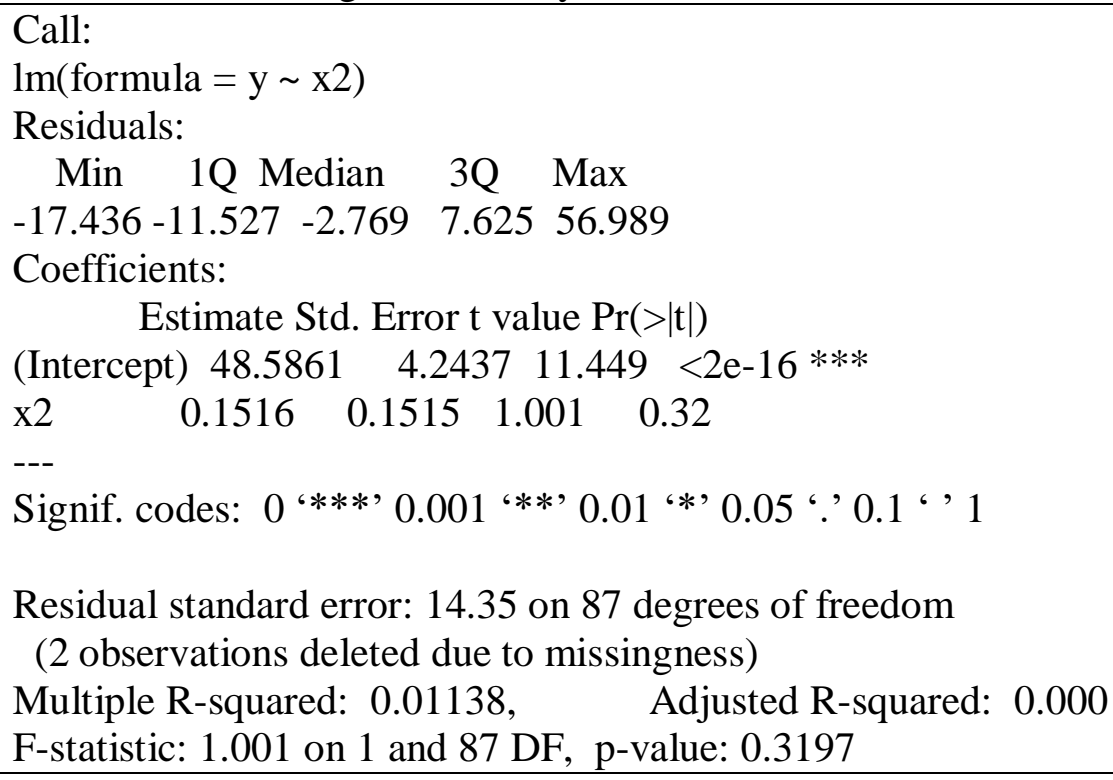

According to the table, the value of the correlation factor between Facebook addiction and self-esteem is 0,01 which represents $1 \%$. It is a weak correlation and the R2 selection coefficient is 0,000 which represents $0 \%$ only. The change in self-esteem is due to internet addiction.

It is also noted that the level of indication is equal to 0,31 which is greater than the level of moral Sig equal to 0,05 . This indicates that there is no difference between selfesteem and Facebook addiction. Therefore, the null hypothesis that Facebook addiction does not affect self-esteem is accepted. The regression line equation between Facebook addiction and Self-esteem can be written as follows: 
$\mathrm{Y}=48,5861+0,1516 \times 2$

Table 11. Regression analysis between the other trend and Facebook addiction

Call:

$\operatorname{lm}($ formula $=\mathrm{y} \sim \mathrm{x} 3)$

Residuals:

Min 1Q Median 3Q Max

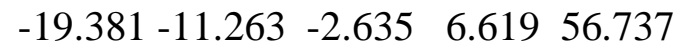

Coefficients:

Estimate Std. Error t value $\operatorname{Pr}(>|t|)$

(Intercept) $45.7710 \quad 4.4464 \quad 10.294<2 \mathrm{e}-16 * * *$

$\begin{array}{lllll}\mathrm{x} 3 & 0.6864 & 0.4235 & 1.621 & 0.109\end{array}$

$---$

Signif. codes: 0 '***' 0.001 '**' 0.01 '*' 0.05 '.' 0.1 ' ' 1

Residual standard error: 14.22 on 87 degrees of freedom

(2 observations deleted due to missingness)

Multiple R-squared: 0.02931, Adjusted R-squared: 0.01815

F-statistic: 2.627 on 1 and $87 \mathrm{DF}$, p-value: 0.1087

According to the table, the value of the correlation factor between Facebook addiction and self-esteem is equal to 0,02 which represents $2 \%$. It is a weak correlation and the $\mathrm{R} 2$ selection coefficient is 0,01 which represents $1 \%$ only. The change in the trend towards the other is due to Facebook addiction.

It is also noted that the level of indication is equal to 0,10 which is greater than the level of moral Sig equal to 0,05 . This indicates that there are no difference between the trend towards the other and Facebook addiction. Accordingly, the null hypothesis that Facebook addiction does not affect the trend towards the other is accepted. The regression line equation between addiction to Facebook and the trend towards the other can be written as follows:

$\mathrm{Y}=45,7710+0,6864 \times 3$

Table 12. Regression analysis between school and professional tendencies and Facebook addiction

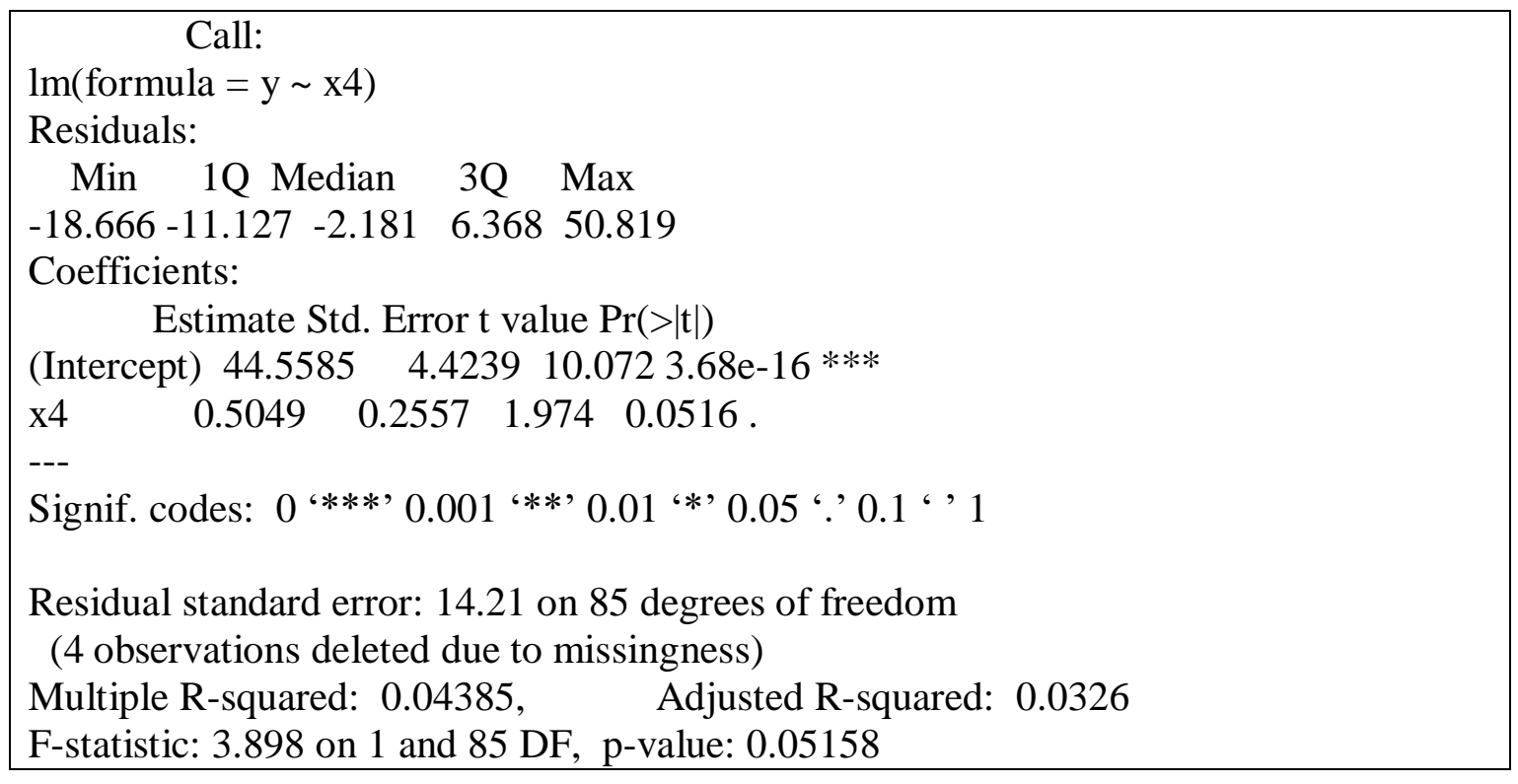


According to the table, the value of the correlation factor between Facebook addiction and school and professional tendencies is equal to 0,04 which represents $4 \%$. It is a weak correlation and the R2 selection factor is 0,03 which represents $3 \%$ only. The change in school and professional tendencies is due to internet addiction.

It is also noted that the level of indication which is 0,05 , is equal to the level of moral Sig. This indicates that there are no difference between school and professional tendencies and Facebook addiction. Therefore the null hypothesis that Facebook addiction does not affect school and professional tendencies is accepted. The regression line equation between Facebook addiction and the trend towards the other can be written as follows: $\mathrm{Y}=44,5585+0,5049 \times 4$

\section{Conclusion}

The current study aimed to identify the impact of Facebook addiction on the identity crisis for the university student. The study was based on a group of questions revolving around How does Facebook's addiction affect a college student's identity crisis? The research sample consisted of 91 students. Based on the results of the study and what was presented through the reliance on statistical data, the researchers reached the following conclusions:

1. The results of the first hypothesis show that:

- The coefficient of correlation between the concept of self and Facebook addiction is statistically significance.

- The coefficient of self-esteem and internet addiction is statistically non-significant.

- The correlation coefficient between trend towards the other and internet addiction is statistically non- significant.

- The correlation coefficient between school and occupational tendencies and Internet addiction is statistically significant.

2. The results of the second hypothesis show that:

- Internet addiction affects the concept of self.

- Internet addiction does not affect self-esteem.

- Internet addiction does not affect the trend towards the other.

- Internet addiction does not affect school and professional tendencies.

\section{Recommendations}

1. Students should be made aware of Facebook's addiction and the consequences of it.

2. Preparing guiding programs to develop the concept of self and psychological identity of the university student.

3. The need to expand and intensify psychological research regarding the identity crisis in individuals.

4. Organizing national and international meetings to discuss the problem of addiction on Facebook.

\section{References}

Aslan, Sevda. (2016). The views of university students regarding internet addiction. Contemporary Educational Researches Journal.vol(6) issue (3), 88-94.

Bas w.Steggink. (w.d). Facebook addiction: Where does it come from? A study based on the Bergen Facebook Addiction Scale. Master Thesis. University of Twente, Department of Communication Science 
Bnirostam, Toura, \& Saberi, Hayedeh.(2017). Study of Loneliness and Identity Styles among Internet Addicted Students in Faculty of Engineering, Islamic Azad University, Central Tehran Branch. Italian Journal of Science \& Engineering. Vol (1) no (2), 82-88.

Chen, Ting-Hsiang \& Hsiao, Ray C \& Lui, Tai-Ling \& Yen, Cheng-Fang.(2019). Predicting effects of borderline personality symptoms and self-concept and identity disturbances on internet addiction, depression, and suicidality in college students: A prospective study.Kaohsiung Journal Medical Sciences.No (35),508-514.

Daniel, Nations.(2019, December,19). What Is Faceboo?[Web log fo.post ].Retrieved from https://www.lifewire.com/what-is-facebook-3486391

Dombrovskis, Aivis.(2016). Identity and an Identity Crisis: The Identity Crisis of FirstYear Female Students At Latvian Untversities And Their Sociodemographic Indicators. Proceedings of the International Scientific Conference. Volume I,,308319.

Dahlena, Sari Marbun \& Azuar, Juliandi \& Sulaiman, Effendi.(2020) The Effect of Social Media Culture and Knowledge Transfer on Performance, Budapest International Research and Critics Institute-Journal, vol (3), no (3), 2513-2520

Dombrovskis, Aivis.(2016). Identity and an Identity Crisis: The Identity Crisis of FirstYear Female Students At Latvian Universities And Their Sociodemographic Indicators. Proceedings of the International Scientific Conference.vol(1), 308-319

E. Cote, James.(2018). The Enduring Usefulness of Erikson's Concept of the Identity Crisis in the 21st Century: An Analysis of Student Mental Health Concerns. Identity: An International Journal Of Theory And Research.1-13

Gwena, Courage \& Willie T. Chinyamurindi \& Marange, Chioneso. (2018). Motives influencing Facebook usage as a social networking site: An empirical study using international students. Independent Research Journal in the Management Sciences, 111

Hsieh, Kuan-Ying \& Hsiao, Ray C \& Yang, Yi-Hsin \& Lee, Kun-Hua \& Yen, ChengFang.(2019). Relationship between Self-Identity Confusion and Internet Addiction among College Students: The Mediating Effects of Psychological Inflexibility and Experiential Avoidance.Intenational Journal of Environment Research and Public Health.

Koo, Hoon Jung \& Kwon, Jung-Hye.(2014). Risk and Protective Factors of Internet Addiction: A Meta-Analysis of Empirical Studies in Korea. Yonsei Med J.vol(55) No(6), 1691-1711.

Mahmood, Shanayyara \& Farooq, Umer. (2014). Facebook Addiction: A Study of BigFive Factors and Academic Performance amongst Students of IUB. Global Journal of Management and Business Research: E Marketing. Vol (14), issue (5), 55-71

Md. Hasnain, Ahamad \& Beplob, Chandra Das.(2018). Facebook Addiction among College Going Students: An Exploratory Study at Patuakhali District in Bangladesh. Social Science and Humanities Journal. Vol (2), issue (10), 617-623

Mana, Alahmad. (2020) , The Effectiveness of Telegram App in Learning English, Budapest International Research and Critics in Linguistics and Education (BirLE) Journal, vol (3), no (3), 1274-1280

Rajesh, Thipparapu \& B. Rangaiah. (2020). Facebook addiction and personality. Heliyon.vol(6). 1-6

Soleymani, Ali \& Farahati, Mehrzad.(2014). The Impact of Excessive Internet Use on Communication Skills and Mental Health in Cafe Internet Users. Int J School Health.1(2),1-6. 